\title{
Simple method for the estimation of solar spectral radiation
}

\author{
Fatima-ezzahra Dahr ${ }^{1 *}$, Omaima El Alani ${ }^{2}$, Abdellah Bah $^{1}$, and Abdellatif Ghennioui ${ }^{3}$ \\ ${ }^{1}$ Thermal and Energy Research Team, University Mohammed V, Ecole Normale Supérieure \\ d'Enseignement Technique, Rabat, Morocco \\ ${ }^{2}$ Laboratory of Signals, Systems and Components, Sidi Mohamed Ben Abdellah Universitu: faculty of \\ science and technology of Fez \\ ${ }^{3}$ Green energy park, Research Institute for Energy and New Energies, Benguerir, Morocco
}

\begin{abstract}
In order to know the energy production of photovoltaic modules, it is necessary to have an idea of the spectral distribution of solar radiation, because each technology is characterized by a different spectral resolution. The objective of this work is to determine the irradiance in ultraviolet, visible, and infrared from the triangular shape of the global solar spectrum for any sky state. According to statistical indicators, the coefficient of determination $\mathrm{R}^{2}$ varies between $97 \%$ and $99 \%$.
\end{abstract}

\section{Introduction}

In our day, electric energy has become a vital aspect of our life, for this reason, several researches launched on a new source that allows producing this energy, yet in a practical, clean, and sustainable way, among these outcomes: the sun. The gathering of solar energy done in two ways, either by a thermal way (CSP) or by solar cells (photovoltaic- PV) and the $\mathrm{PV}$ is one of the most available technologies today.

In order to be able to capture the maximum solar radiation, two concepts must tackle: firstly, the development of reception materials, and secondly, the efficient knowledge of solar radiation and all the atmospheric components that modify it. With this objective, this project focuses on the spectral distribution of solar radiation, which is considered as a distribution of solar radiation as a function of wavelength, which is a parameter that contains several pieces of information about the state of the earth's sky.

Several expressions used related to solar spectral such as: The solar irradiance (SI) is the emitted light energy from the sun measured at the earth level. The spectral solar irradiance (SSI) W. $\mathrm{m}^{-2} \cdot \mathrm{nm}^{-1}$ is the wavelength-dependent energy, the total solar irradiance sum (TSI) $\mathrm{W} . \mathrm{m}^{-2}$ is the SSI integrated over the whole spectrum and is generally made up of visible and infrared radiation with the small proportion of ultraviolet.

The descending spectral solar irradiance is divided into several parts, each having an influence on a specific domain, for example:

- In agriculture, agronomists are interested in photosynthetically active PAR radiation [400 to $700 \mathrm{~nm}$ ]

* Corresponding author: fatimaezzahra.dahr@um5s.net.ma 
- In architecture, architects must estimate the visible radiation for natural lighting in buildings [380 to $780 \mathrm{~nm}$ ]

- In health: epidemiologists may need irradiance in the UV-A range

- In power generation, each photovoltaic technology has a different spectral response see figure 1, the spectral response of the PV cell will describe the efficiency of the panel to transform the spectral solar irradiance into electricity according to the given PV technology and the given conditions.

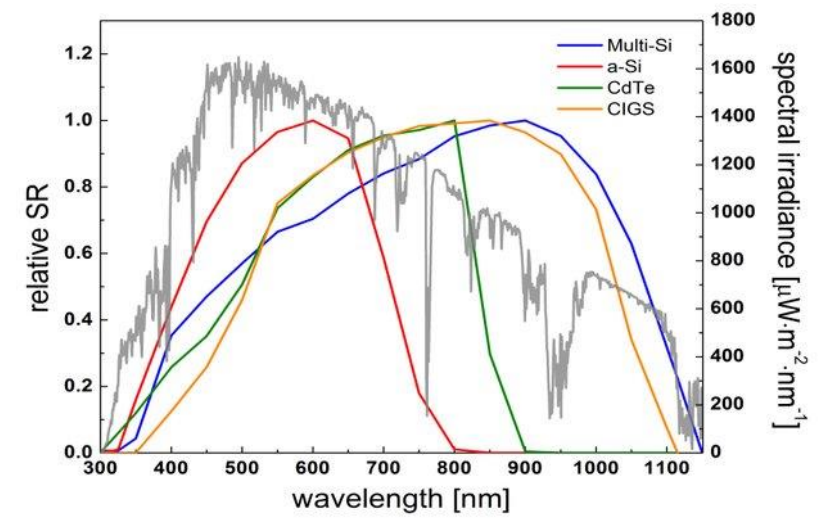

Fig. 1. Spectral response for different technologies (Multi-Si, a-Si, CdTe, CIGS) under STC condition $\left(25^{\circ} \mathrm{C}, 1000 \mathrm{w} / \mathrm{m}^{2}, \mathrm{AM} 1.5\right)$ with AM1.5G spectrum (gray) [1]

The broad integration of photovoltaic technology in recent years makes the estimation of the spectral distribution of solar radiation essential, as it allows us to know the photovoltaic production.

The complex part of the forecast or estimation is that the solar spectrum is sensitive to atmospheric factors, in general, incident radiation goes through an attenuation pro-cess that contains both absorption and scattering, the first is a source of heat in the atmosphere and the second is responsible for diffuse radiation.

The imposed problem is that spectral measurements in a given site are expensive and difficult to obtain. The solution is to extract from the available data is easy to find such as Global Horizontal Irradiance $\mathrm{G}_{0}$.

Several studies indicate that the UV part of the solar spectrum affects the electrical part of photovoltaic modules. As well as the prediction of the spectral distribution, it is possible to predict the production of the photovoltaic modules of the different technologies, because each technology characterized by a different spectral response as mentioned before.

This method is based on a simple algorithm to estimate the spectral distribution, of total radiation and solar radiation at ground level using spectral measurements from Uccle, Belgium, developed by Crommelynck and Joukoff [2].

\section{Methodology}

In this project, a simple method based on the normal shape of the solar spectral proposed in order to estimate the global solar spectral irradiance, by using the simple linear regression, and especially the UV irradiance, which requires as input only one meteorological parameter.

\subsection{Study Site}

The radiation data used in this study measured at the Green Energy Park (GEP) weather station (figure 2). 


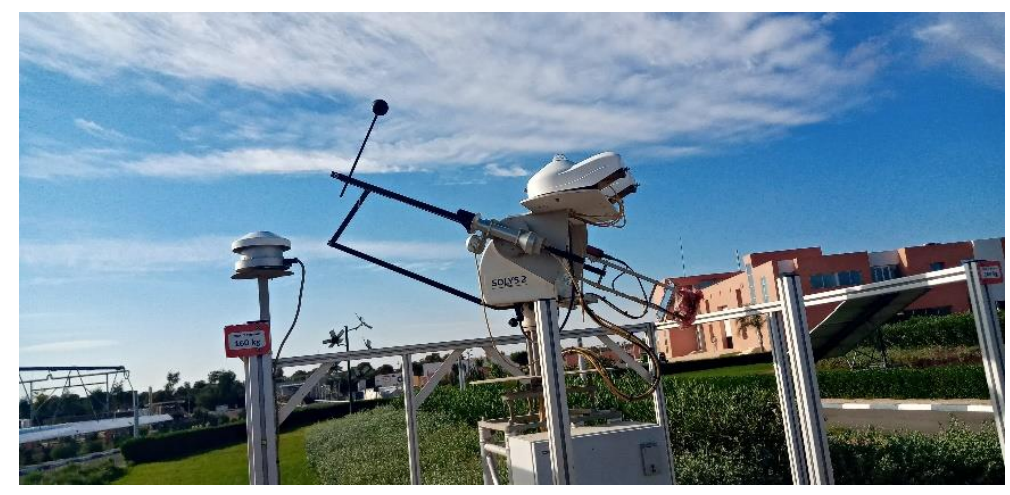

Fig. 2. The weather station at GEP.

The meteorological data that we can collect from the weather station are as follows: air temperature, relative humidity, precipitation, wind speed, horizontal global irradiation, normal direct irradiation, horizontal diffuse irradiation, solar spectrum, etc.

Table 1. Description of the spectral irradiance datasets and collection sites

\begin{tabular}{|l|l|}
\hline Latitude $\left({ }^{\circ}\right)$ & $32.12^{\circ} \mathrm{N}$ \\
\hline Longitude $\left(^{\circ}\right)$ & $7.94^{\circ} \mathrm{E}$ \\
\hline Altitude $(\mathrm{m})$ & 450 \\
\hline Average site temperature $\left({ }^{\circ} \mathrm{C}\right)$ & 21.3 \\
\hline Average site pressure $(\mathrm{mbar})$ & 962.6 \\
\hline Start wavelength $(\mathrm{nm})$ & 286 \\
\hline End wavelength $(\mathrm{nm})$ & 1117 \\
\hline
\end{tabular}

The world map of Köppen-Geiger [3] concerning the climatic condition consider the climate at Benguerir (Cwa) is classified as Warm temperature/ winter dry/ hot summer.

\subsection{Description of the new method}

Spectral data are hardly available for a specific area, for this reason several algorithms have been developed, based on solar irradiance at ground level or on other climatic parameters, in order to estimate the solar spectrum and therefore reduce this lack. among the problem when estimating solar spectrum using a model is the size of the database used, for each instant the solar spectrum contains 800 points (in our case between 300 and $1100 \mathrm{~nm}$ ), so for a only year we will have a matrix of $800 * 52560$ if the data is take every 10 min. This work presents, firstly, a technique that represents the solar spectrum under a triangular representation and thus reduce the number of data, secondly, a method of estimation of the spectral irradiance. This new method based on the determination of the triangular shape that frames the solar spectrum, as shown in Figure 3; the coordinates of the three points are as follows:

-Point 1: x1=300 nm;

-Point 2: x2= $481 \mathrm{~nm}$;

$$
y 1=0 \mathrm{mw} / \mathrm{m}^{2} . \mathrm{nm}
$$

-Point 3: x3=1100 nm;

$$
\begin{aligned}
& y 2=A 1 * G_{0} \\
& y 3=A 2 * G_{0}
\end{aligned}
$$


y2 and y3 correspond successively to the irradiance of the maximum point and end point of the solar spectrum, are determined from a correlation between the irradiance $G_{0}$ and $\max$ point of the solar spectra for point 2 (see (1)) and end point for point 3 (see (2)).

The interest of this method is that it allows us to easily determine the shape of our solar spectrum using only one parameter, G0, and from this shape, we can have an idea about 10 wavelengths of the spectrum and also we can have information about the irradiance in UV, Visible, and IR, as well as the estimation with neural networks and much faster because we can base ourselves just for the 3 points.

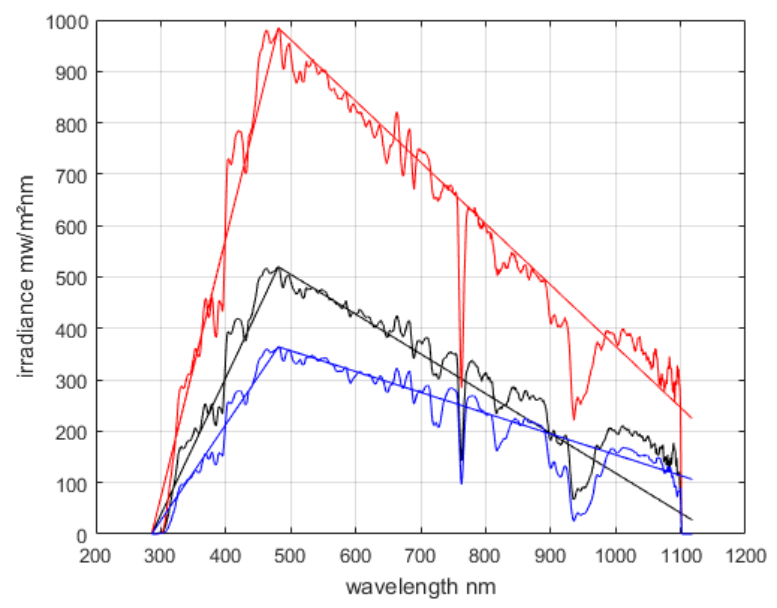

Fig. 3. The triangular shape and the solar spectrum for different times

Integrating raw data is often a bad decision, because in most cases raw data is unreliable and noisy, which can lead to erroneous results. In order to minimize the impact of the nonnegligible fluctuation of a solar spectrum, a pre-processing would be the best solution.

This method only needs the global horizontal irradiation $\mathrm{G}_{0}$ as main input, which is widely available for any place and time, either measured or calculated or downloaded from several open source databases, by means of a Simple Linear Regression SLR one can extract the maximum point and end point of the solar spectrum which corresponds successively to 481 and $1100 \mathrm{~nm}$, and the estimated starting point $0 \mathrm{mw} / \mathrm{m}^{2} . \mathrm{nm}$, so from the 3 points one has the possibility to determine the two equations of the triangular form TS:

$$
\begin{array}{lll}
T S_{1}=\alpha_{1} \times \lambda_{1}+\beta & \text { with } & \alpha 1>0 ; 300 \mathrm{~nm} \leq \lambda_{1} \leq 481 \mathrm{~nm} \\
T S_{2}=\alpha_{2} \times \lambda_{2}+\beta & \text { with } & \alpha 2<0 ; 481 \mathrm{~nm}<\lambda_{2} \leq 1100 \mathrm{~nm}
\end{array}
$$

We have also intuitively chosen ten wavelengths $(315,330,430,481,524,590,690,815$, 900 , and $966 \mathrm{~nm}$ ) that represent the intersection between the two curves as shown in figure 5 , then from TS we have the information about this 10 points of the solar spectrum and which are close to the measured one (table 2).

This simple method also gives us the irradiance of each part of the solar spectrum (UV, VIS, and IR) with high accuracy. 


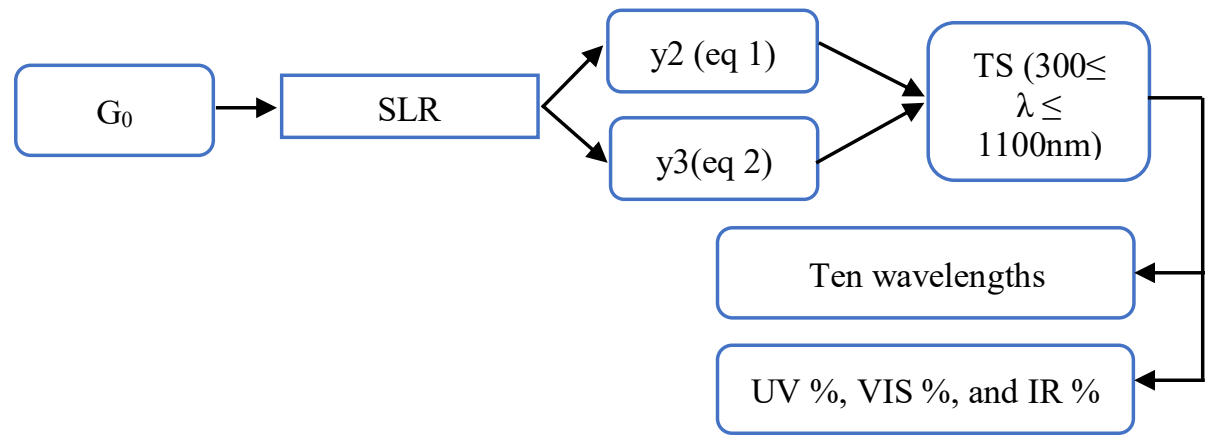

Fig 4. The methodology followed for this model

Figure 5 represent the localisation of the wavelength that we can extract from the TS

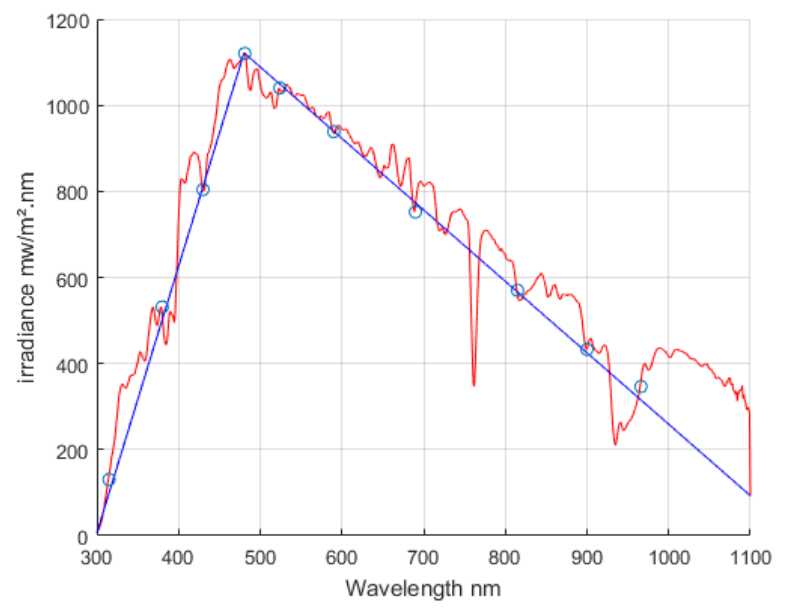

Fig .5. The location of the ten wavelengths chosen in this study on the measured solar spectrum and the TS

\section{Comparison by measurement}

Statistical indicators are indices that are widely used by researchers to compare between models. The indices that used in this study are:

Mean Bias (MBE):

The root mean square error (RMSE):

$$
M B E=\sum_{i=1}^{n} \frac{\left(\hat{y}_{i}-y_{i}\right)^{2}}{n}
$$

$$
R M S E=\sqrt{\sum_{i=1}^{n} \frac{\left(\hat{y}_{i}-y_{i}\right)^{2}}{n}}
$$

In addition, the coefficient of determination $\left(\mathrm{R}^{2}\right)$ :

$$
R^{2}=\frac{\sum_{i=1}^{n}\left(\hat{y}_{i}-\overline{\hat{y}}_{i}\right)^{2} \times \sum_{i=1}^{n}\left(y_{i}-\overline{y_{i}}\right)^{2}}{\sqrt{\sum_{i=1}^{n}\left(\hat{y}_{i}-\overline{\hat{y}}_{i}\right)^{2} \times \sum_{i=1}^{n}\left(y_{i}-\overline{y_{i}}\right)^{2}}}
$$


From table 2, we can observe that the $\mathrm{R}^{2}$ for all points are high and tend towards one, but for the other indicators (RMSE (4) and $\operatorname{MBE}(3)$ ), we can say that there is a small difference in the UV part in due to the large fluctuation of the solar spectrum due to absorption by the ozone layer,

Table. 2 Statistical indicators of the ten chosen wavelengths

\begin{tabular}{|c|l|l|l|}
\hline Wavelengths & MBE $\%$ & RMSE $\%$ & $\mathrm{R}^{2}$ \\
\hline $\mathbf{3 1 5}$ & 18.66 & 4.32 & 0.961 \\
\hline $\mathbf{3 3 0}$ & 20.8 & 4.56 & 0.996 \\
\hline $\mathbf{4 3 0}$ & 61.67 & 7.8 & 0.996 \\
\hline $\mathbf{4 8 1}$ & 3.87 & 1.96 & 0.999 \\
\hline $\mathbf{5 2 4}$ & 2.46 & 1.56 & 0.996 \\
\hline $\mathbf{5 9 0}$ & 5.99 & 2.447 & 0.991 \\
\hline $\mathbf{6 9 0}$ & 3.77 & 1.94 & 0.999 \\
\hline $\mathbf{8 1 5}$ & 20.29 & 4.50 & 0.980 \\
\hline $\mathbf{9 0 0}$ & 9.40 & 3.06 & 0.988 \\
\hline $\mathbf{9 6 6}$ & 13.70 & 37.02 & 0.971 \\
\hline
\end{tabular}

To ensure the reliability of our method, we compare the Global horizontal irradiance $\mathrm{G}_{0}$ with the weighted average of the triangular shape as shown in Figure 6. In order to calculate the weighted average each number of the triangular shape is multiplied by its corresponding wavelength in order to have irradiance with a unit of $\mathrm{w} / \mathrm{m}^{2}$.

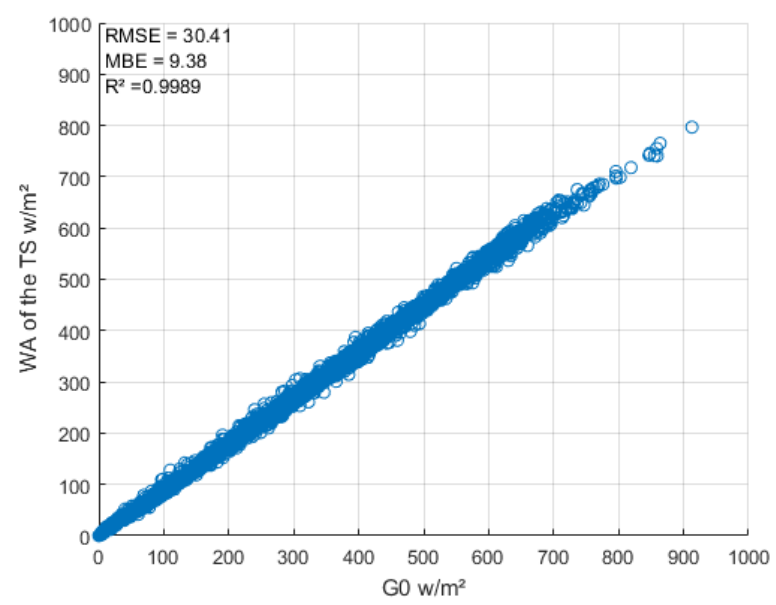

Fig. 6. Difference between the Global Horizontal Irradiance $\mathrm{G}_{0}$ and the weighted average of the triangle shape

Several Methods have been carried out for the estimation of the spectral distribution of solar radiation [4 -7]. This method is applicable for all wavelengths; this study is carried out mainly for UV irradiance, as this wavelength range is known by its non-negligible influence on silicon-based photovoltaic modules.

With only $\mathrm{G}_{0}$, we can also extract the irradiation for ultraviolet UV, visible VIS, infrared IR, with high accuracy for all sky conditions (figure. 7). 


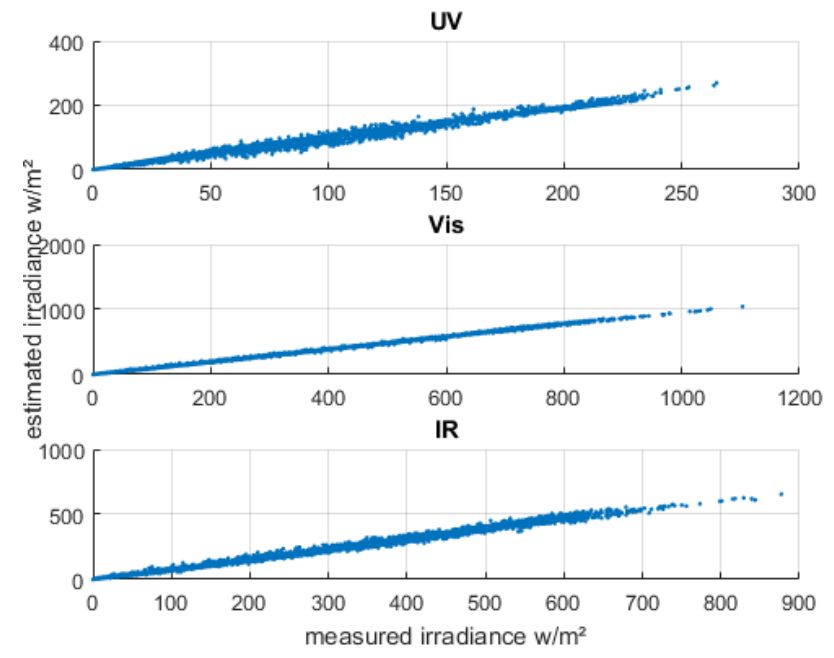

Fig. 7. Difference between the measured and estimated irradiance for the three parts of the solar spectral irradiance (Ultraviolet, Visible, Infrared)

Table. 3 statistic indicators for Difference between the measured and estimated irradiance for the three parts of the solar spectral irradiance (Ultraviolet, Visible, Infrared)

\begin{tabular}{|l|l|l|l|}
\cline { 2 - 4 } \multicolumn{1}{c|}{} & Ultraviolet (UV) & Visible (VIS) & Infrared (IR) \\
\hline MBE \% & 10,31 & 5,96 & 7,09 \\
\hline RMSE \% & 23,62 & 7,17 & 11.72 \\
\hline R $^{2}$ & 0.97 & 0.99 & 0.98 \\
\hline
\end{tabular}

For a better visibility of the difference between the two spectra, statistical indicators are calculated. The new method as shown in table 3 gives good results.

As we see in the figure 7 and the table 3 , we have a high correlation between measured and estimated values of the irradiance.

\section{Discussions and conclusion}

A new methodology based on a single parameter to estimate the shape of the global solar spectrum and from this shape we can have the irradiance for ten wavelengths with great precision ( $\mathrm{R}^{2}$ between 0.96 and 0.99 ) the method has been evaluated compared to the measured spectrum in order to estimate and reconcile their performance. Therefore, validation is done experimentally.

From experimental information collected in Benguerir (Morocco) the climate is classified as (Cwa) hot temperature / dry winter / hot summer. This method is valid for any type of sky because the data used is not sorted according to the clarity index.

The main advantage of the proposed method is that the environmental parameter required as input data for the model is usually available in meteorological databases. This model helps to reliably estimate the impact of the solar spectrum on the performance of photovoltaic materials, and especially studies regarding the discoloration of the ethylene vinyl acetate (EVA) layer, when spectroradiometric measurements are not available. 


\section{References}

1. H. Liu et al., The Impact of Haze on Performance Ratio and Short-Circuit Current of PV Systems in Singapore," IEEE J. Photovoltaics, vol. 4, no. 6, pp. 1585-1592, Nov. (2014)

2. D. Crommelynck and A. Joukoff, A simple algorithm for the estimation of the spectral radiation distribution on a horizontal surface, based on global radiation measurements, Solar Energy, vol. 45, no. 3, pp. 131-137, (1990)

3. M. Kottek, J. Grieser, C. Beck, B. Rudolf, and F. Rubel, World Map of the KöppenGeiger climate classification updated, metz, vol. 15, no. 3, pp. 259-263, Jul. (2006)

4. J. del Campo-Ávila, M. Piliougine, R. Morales-Bueno, and L. Mora-López, A data mining system for predicting solar global spectral irradiance. Performance assessment in the spectral response ranges of thin-film photovoltaic modules, Renewable Energy, vol. 133, pp. 828-839, Apr. (2019)

5. W. W. Nyamsi, B. Espinar, P. Blanc, and L. Wald, A new approach for estimating operationally the spectral distribution of surface solar irradiance: preliminary results, 8 (2015)

6. Torres-Ramírez, D. Elizondo, B. García-Domingo, G. Nofuentes, and D. L. Talavera, "Modelling the spectral irradiance distribution in sunny inland locations using an ANN-based methodology," Energy, vol. 86, pp. 323-334, Jun. (2015)

7. Habte, M. Sengupta, C. A. Gueymard, R. Narasappa, O. Rosseler, and D. M. Burns, "Estimating Ultraviolet Radiation From Global Horizontal Irradiance," IEEE Journal of Photovoltaics, vol. 9, no. 1, pp. 139-146, Jan. (2019) 